\title{
Theoretical and Experimental Investigation of Characteristics of Single Fracture Stress-Seepage Coupling considering Microroughness
}

\author{
Shengtong Di, ${ }^{1,2}$ Chao Jia, ${ }^{1}$ Weiguo Qiao,, ${ }^{2,3}$ Weijiang Yu, ${ }^{1}$ and Kang $\mathrm{Li}^{1}$ \\ ${ }^{1}$ School of Civil Engineering, Shandong University Jinan, Shandong 250061, China \\ ${ }^{2}$ Key Laboratory of Disaster Prevention and Reduction of Civil Engineering in Shandong Province, Qingdao, Shandong 266590, China \\ ${ }^{3}$ School of Civil Engineering and Architecture, Shandong University of Science and Technology Qingdao, Shandong 266590, China \\ Correspondence should be addressed to Chao Jia; jiachao@sdu.edu.cn
}

Received 7 April 2017; Accepted 28 May 2017; Published 19 July 2017

Academic Editor: Mohammed Nouari

Copyright (C) 2017 Shengtong Di et al. This is an open access article distributed under the Creative Commons Attribution License, which permits unrestricted use, distribution, and reproduction in any medium, provided the original work is properly cited.

\begin{abstract}
Based on the results of the test among the joint roughness coefficient (JRC) of rock fracture, mechanical aperture, and hydraulic aperture proposed by Barton, this paper deduces and proposes a permeability coefficient formula of single fracture stress-seepage coupling considering microroughness by the introduction of effect variables considering the microparticle size and structural morphology of facture surface. Quasi-sandstone fracture of different particle size is made by the laboratory test, and the respective modification is made on the coupled shear-seepage test system of JAW-600 rock. Under this condition, the laboratory test of stressseepage coupling of fracture of different particle size is carried out. The test results show that, for the different particle-sized fracture surface of the same JRC, the permeability coefficient is different, which means the smaller particle size, the smaller permeability coefficient, and the larger particle size, the larger permeability coefficient; with the increase of cranny hydraulic pressure, the permeability coefficient increases exponentially, and under the same cranny hydraulic pressure, there is relation of power function between the permeability coefficient and normal stress. Meanwhile, according to the theoretical formula, the microroughness coefficient of the fractures with different particle size is obtained by the calculation, and its accuracy and validity are verified by experiments. The theoretical verification values are in good agreement with the measured values.
\end{abstract}

\section{Introduction}

At present, with the continuous development of national economic construction, as a result of the unceasing extending in such project fields as the mining, tunnels, water conservancy and hydropower, nuclear waste repository, and foundation engineering, the development level of underground space is increasing day by day, and the confronted engineering problems are becoming more and more complicated. As a complex media in almost all geotechnical engineering fields, with the jointed rock mass, which exists in a large number of fractures and microfractures and other joints, under the action of geological structure and human activities, these joints not only reduce the strength of rock mass greatly, but also constitute an important channel for groundwater to flow in rock mass. For the seepage hydraulic pressure, whose main source is the underground confined water, under the different circumstances of water storage environment and stress, it has different external impact force and permeability effect on the crack structure of the rock mass, and the existing structures of the fractures are destroyed; and the change of the fissure structure will cause the change of the permeability channel, which will affect the stress distribution of the rock itself, and the new secondary cracks will be generated further. The interaction in which the seepage field of fissured rock mass can be influenced by the stress environment and the stress distribution can be influenced by changes of the seepage field is called stress-seepage coupling. The coupling feature is one of the important features of rock mass mechanics. After the dam-break of the Malpasset Dam in France (1959) and the 
landslide of the Vaiont Dam in Italy (1963), the problem gradually draws people's attention and a large number of studies [1] are carried out.

As one of the key fundamental topics in the research field of geotechnical engineering, single fracture stress-seepage coupling has been studied through a lot of theoretical and experimental researches from many aspects by many research scholars at home and abroad. Bandis et al. establish the relationship between the fracture closure and stress, respectively, through the experiment [2-5]. Barton and Choubey divide all the joint surfaces into 10 levels from smooth to rough according to their roughness by analyzing the undulating roughness of 136 joint surfaces, where JRC $=0-20$, and present the typical roughness profile [6]. Barton proposes the relationship among the joint roughness coefficient (JRC), the hydraulic aperture, and the mechanical aperture [7] by a large number of experiments, but the formula can only be limited to the occasions where the mechanical aperture is larger than the hydraulic aperture, and the unit of them is micron-sized. Lomize et al. have carried out groundbreaking experiments on the permeability law of fracture and establish the cubic law that the seepage flux and the fracture aperture are cubically proportioned [8-10], which lay the foundation of the permeability law of fracture. After studying the microfracture $(10-100 \mu \mathrm{m})$ and the extreme microfracture $(0.25-4.3 \mu \mathrm{m})$, Romm proposed that the cubic law will always be established, as long as the fracture aperture is bigger than $0.2 \mu \mathrm{m}$ [9]. However, the real fractures are rough, and then, many researchers have done a lot of experiments on the roughness of fracture surfaces, and the cubic law was amended [11-17]. In the case of single fracture stressseepage coupling, many scholars establish the relationship between the permeability coefficient and the stress from the perspective of the seepage experiments or the fracture deformation [18-30]. This paper summarizes the domestic and foreign scholars' research results about the single fracture roughness, the relationship between the fracture deformation and the stress, and stress-seepage coupling experiments that all of them basically adopt the natural test pieces splitting or the artificial normal fracture and rarely involve the studies considering the differences in features and laws of the seepage capability due to the differences of microparticles and their structure of the surface.

Therefore, in order to investigate the effect of the microparticle size and structure on the seepage capability of fracture, this paper tries to deduce the theoretical formula of the permeability coefficient of single fracture surfaces under the coupling of normal stress and the cranny hydraulic pressure by considering the influencing factors of the microparticle size of fracture surfaces and introducing the microroughness coefficient on the basis of results obtained by Barton in 1982 through lots of experiments. Then the fractures of different particle size are prepared in the laboratory based on the same JRC for the use of simulating the different microroughness; the stress-seepage coupling tests are carried out, respectively, to obtain the permeability coefficient of fracture surfaces with different particle size, and corresponding microroughness coefficients are calculated. Finally, the accuracy and validity of the seepage theoretical formula considering the influence of microroughness are verified through parallel tests. This study is a supplement to the theory of fracture stress-seepage coupling for the jointed rock mass, and it also has a great theoretical guiding significance for the phenomenon of microfracture seepage in geotechnical engineering field, which will be the important reference for the intensive study of the seepage law of microfracture surfaces in the future.

\section{Theory of Stress-Seepage Coupling considering Microroughness}

It is assumed that the flow condition of water in the factures is laminar flow; the water is incompressible and viscous in the process; the water flow only seeps along fracture surfaces without other loss of flow; the cranny hydraulic pressure acting on fracture surfaces is regarded as the average head pressure at the inlet and outlet ends.

According to the constitutive equation of fracture deformation of jointed rock mass [4], the fracture deformation $\Delta u$ can be expressed as

$$
\Delta u=\mu_{0}\left(1-e^{-\sigma_{f} / K_{n}}\right),
$$

where $\mu_{0}$ is the maximum compression deformation of fractures, $\sigma_{f}$ is the normal load acting on fracture surfaces, and $K_{n}$ is the normal stiffness of the fracture surface.

Therefore, the mechanical aperture of the fracture surface $\mu$ is

$$
\mu=\mu_{0} e^{-\sigma_{f} / K_{n}}
$$

In 1982, considering the condition of normal stress loading, Barton obtains the relationship among the equivalent hydraulic aperture, the mechanical aperture, and the joint roughness coefficient (JRC) based on a large number of test results [7]:

$$
a_{w}=\frac{\mu^{2}}{\mathrm{JRC}^{2.5}} .
$$

However, the formula only involves the macroroughness coefficient of the fracture, strictly speaking, the waviness of the fracture, without considering the viscous effect of the geometric size and combination mode of the microparticles of the fracture surface on the water flow. Some scholars at home and abroad have proposed some modified formulas for the Barton model, but all of them only consider the occasions of different JRC but not the influence of the particle size and distribution of fracture particles on the seepage capacity of fracture surfaces. The microroughness is the minimum level of rough and undulation form of the fracture surface to reflect the sublevel geometric characteristics on the surface of the peak valley and the concrete distribution manifestation of mineral particles or tiny crystals on joints surface, while the essential features of microroughness depend on the component, structure size, crystal form, and combination of the mineral crystals of the rock on the fracture surface and its exposure on the fracture surface [31]. 
Introduce the microroughness coefficient $C_{m}$ that expresses the microgeometric characteristics of the fracture surface to measure the influence of different particle size and distribution of fracture particles of the fracture surface on the viscous effect of the water flow, and the value can be calculated by the stress-seepage coupling test. Among them, $C_{m}=R_{m} / R_{0}, R_{m}$ is the microroughness of the fracture surface of jointed rock mass used in the test, and the value relies on the particle size and distribution of the component particles of fracture; $R_{0}$ is the microroughness of test piece in the experiments adopted by Barton and regarded as the datum reference; $R_{m}$ and $R_{0}$ both are functions related to the microparticle size and distribution of the fracture surface.

Therefore, formula (3) can be transformed into

$$
a_{w}=\frac{\mu^{2} C_{m}}{\mathrm{JRC}^{2.5}}
$$

Substitute the mechanical aperture formula (2) into formula (4) and get the formula

$$
a_{w}=\frac{\mu_{0}^{2} C_{m} e^{-2 \sigma_{f} / K_{n}}}{\mathrm{JRC}^{2.5}} .
$$

Substitute formula (5) into the cubic law, and then get the permeability coefficient formula that considers the microroughness as follows:

$$
k_{f}=\frac{g a_{w}{ }^{2}}{12 \nu}=\frac{g \mu_{0}{ }^{4} C_{m}{ }^{2}}{12 \nu \mathrm{RC}^{5}} e^{-4 \sigma_{f} / K_{n}},
$$

where $\mu_{0}$ is the initial aperture of the fracture surface; $K_{n}$ is the normal stiffness of the fracture surface; $\sigma_{f}$ is the normal load acting on fracture surfaces; $C_{m}$ is the microroughness coefficient related to the particle size and distribution of the fracture surface; $v$ is the viscosity coefficient of water flow movement; JRC is the joint roughness coefficient of rock fracture.

Formula (6) can be analyzed as follows: the permeability coefficient of the fracture is equal to the product of $g \mu_{0}{ }^{4} C_{m}{ }^{2} / 12 \nu \mathrm{JRC}^{5}$, the initial permeability coefficient term considering JRC and microroughness, and $\exp \left(-4 \sigma_{f} / K_{n}\right)$, and the impact term decreased in the negative exponential law. Among them, when the value of $\sigma_{f}$ is smaller than $K_{n}$, there is the approximate simplification $\sigma_{f} / K_{n}=\Delta u / \mu_{0}=\varepsilon_{n}$ [5]; therefore, formula (6) can be written into

$$
k_{f}=\frac{g a_{w}{ }^{2}}{12 \nu}=\frac{g \mu_{0}{ }^{4} C_{m}{ }^{2}}{12 \nu \mathrm{RC}^{5}} \exp \left(-4 \varepsilon_{n}\right) .
$$

In the formula, $\varepsilon_{n}$ is the normal deformation of the changes of permeability coefficient caused by the fracture surface.

In order to determine the amount of deformation of the fracture surface under the combined action of normal load and cranny hydraulic pressure, it is possible to calculate the change of fracture aperture more easily through indoor experiment; therefore, the following calculation is performed. Figure 1 shows the stress-seepage coupling calculation model

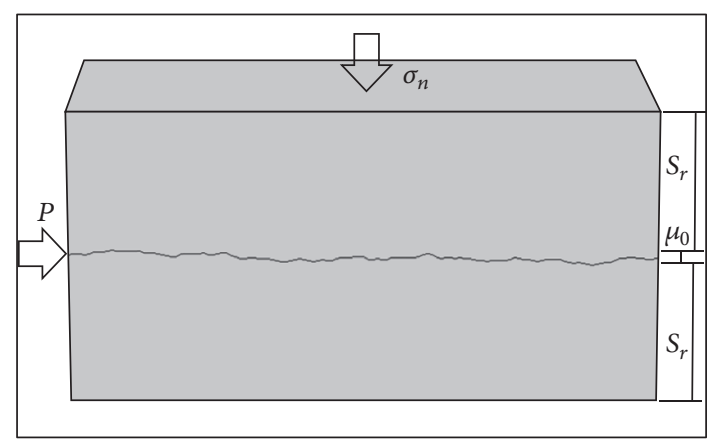

FIgURE 1: The calculation model of single fracture under stressseepage coupling.

of fracture rock masses under the condition of the normal load $\sigma_{n}$. When adding the hydraulic pressure $P$ along the side of the fracture, the total displacement of the jointed rock mass $\left(\Delta u_{t}\right)$ is equal to the sum of the fracture displacement $\left(\Delta u_{f}\right)$ and the displacement of the rock $\left(\Delta u_{r}\right)$. The fracture displacement $\Delta u_{f}$ can be expressed as

$$
\Delta u_{f}=\Delta u_{t}-\Delta u_{r} .
$$

Supposing the rock thickness is $S_{r}$ and the fracture aperture is $\mu_{0}$, the strain can be expressed as

$$
\Delta u_{f}=\left(2 S_{r}+\mu_{0}\right) \varepsilon_{t}-2 S_{r} \varepsilon_{r},
$$

where $\varepsilon_{t}$ and $\varepsilon_{r}$ are the strain of rock masses and rocks, respectively.

The strain of rock masses can be expressed as

$$
\varepsilon_{t}=\frac{\sigma_{n}}{E_{t}},
$$

where $E_{t}$ is the elastic modulus of monolithic rock mass that includes the fractures. Considering the effect of the cranny hydraulic pressure, both of it and the normal load can lead to the compressive deformation of rocks; therefore, the strain of rocks can be expressed as

$$
\varepsilon_{r}=\frac{\left(\sigma_{n}+\Delta p_{0}\right)}{E_{r}} .
$$

In formula (11), $E_{r}$ is the elastic modulus of rocks. Substitute formulas (10) and (11) into formula (9); we can get the formula

$$
\Delta u_{f}=\frac{\left(2 S_{r}+\mu_{0}\right) \sigma_{n}}{E_{t}}-\frac{2 S_{r}\left(\sigma_{n}+\Delta p_{0}\right)}{E_{r}} .
$$

After the arrangement, the formula is

$$
\Delta u_{f}=\left(\frac{2 S_{r}+\mu_{0}}{E_{t}}-\frac{2 S_{r}}{E_{r}}\right) \sigma_{n}-\frac{2 S_{r} \Delta p_{0}}{E_{r}} .
$$

The fracture strain can be described as

$$
\varepsilon_{n}=\frac{\Delta u_{f}}{\mu_{0}}=\left(\frac{2 S_{r}+\mu_{0}}{\mu_{0} E_{t}}-\frac{2 S_{r}}{\mu_{0} E_{r}}\right) \sigma_{n}-\frac{2 S_{r} \Delta p_{0}}{\mu_{0} E_{r}} .
$$


Substitute formula (14) into formula (7); under the coupling of normal load and the seepage pressure, after comprehensively considering the joint roughness coefficient JRC and the microroughness coefficient $C_{m}$, the obtained formula of permeability coefficient $k_{f}$ of the single fracture of the jointed rock mass is

$$
\begin{aligned}
k_{f} & =\frac{g a_{w}{ }^{2}}{12 \nu}=\frac{g \mu_{0}{ }^{4} C_{m}{ }^{2}}{12 \nu \mathrm{RC}^{5}} \\
& \cdot \exp \left[\frac{8 S_{r} \Delta p_{0}}{\mu_{0} E_{r}}-\left(\frac{8 S_{r}+4 \mu_{0}}{\mu_{0} E_{t}}-\frac{8 S_{r}}{\mu_{0} E_{r}}\right) \sigma_{n}\right] .
\end{aligned}
$$

Formula (15) is the theoretical mathematical model of single fracture stress-seepage coupling considering microroughness, which comprehensively considers the compressive deformation of the stress and seepage pressure on the fracture surface, the joint roughness coefficient of the fracture surface (JRC), and the microroughness coefficient of the fracture surface $R_{m}$. At the same time, parameters $E_{t}, E_{r}$, and $\mu_{0}$ can be calculated by the indoor tests, and they have the general applicability.

In the calculation, because formula (3) is the result of data fitting law based on a large number of experiments by Barton, and it can only be used in the micron-sized units, in the real calculation, it is necessary to change the unit; that is, in this step of calculation, the unit should be transformed into micron-sized unit firstly and changed into the standard unit after the calculation.

\section{Preparation of Fracture Test Pieces and Experimental Work}

3.1. Test Principles. We use the steel template of the same JRC to pour the test pieces of different particle-sized fracture surface, adopt the same manufacturing process, and carry on maintenance in the same maintenance environment in order to ensure the same macroroughness. Based on the analysis of the permeability coefficient in the test results, the microroughness coefficient of different-particle-sized fracture surfaces can be calculated to analyze the effect of microroughness on the seepage capability of fracture surfaces.

The following conversion formula is used to calculate the measured permeability coefficient of the fracture surface; then the permeability coefficient is substituted into formula (15), and the microroughness of particle-sized fracture surfaces can be calculated. The fracture aperture is expressed as the hydraulic equivalent aperture. The calculation equation of the seepage flow of the single fracture surface is shown in the following equation:

$$
q=\frac{g w a_{w}{ }^{3} \Delta H}{12 \nu L} .
$$

According to the basic seepage theory of fracture rock mass, the permeability coefficient of the fracture of jointed rock mass can be expressed as

$$
k_{f}=\frac{g a_{w}^{2}}{12 v} .
$$

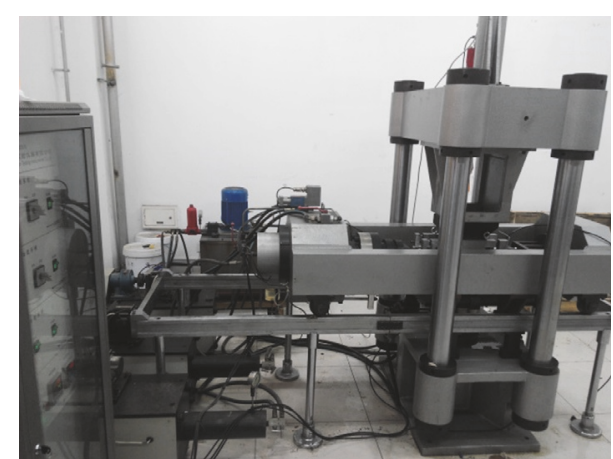

FIGURE 2: The stress-seepage coupling test system.

Convert formula (16) and substitute it into formula (17); we can get the following formula through conversion:

$$
k_{f}=\sqrt[3]{\frac{g L^{2} q^{2} \gamma_{\omega}{ }^{2}}{12 \nu w^{2} \Delta p^{2}}},
$$

where $k_{f}$ is the permeability coefficient of the fracture test piece; $q$ is the seepage flow through the fracture surface; $L$ is the path length of the fracture surface where the liquid flows; $\nu$ is the dynamic viscosity of water; $\gamma_{\omega}$ is the severity of water; $w$ is the width of the fracture surface where the liquid flows; $\Delta p$ is the difference of seepage pressure of the inlet and outlet ends.

3.2. Test Instruments and Systems. The coupled shear-seepage test system of JAW-600 rock is adopted in the test. As the most advanced rock shear-seepage test system in China, it consists of the loading system, the confining pressure system, the hydraulic system, the seepage box, the control system, the computer system, and other components. It can realize the normal servo controlled test under the conditions of constant normal stress, constant normal displacement, and constant normal stiffness; the seepage test under different seepage pressures; the test of closure stress-seepage coupling under the conditions of different seepage pressures; and the test of shear stress-seepage coupling under the conditions of different seepage pressures.

The site experiment picture is shown in Figure 2.

Considering the purpose of the test, it only involves the normal load, regardless of the shear loading module; at the same time, corresponding transformation of the seepage box is done, the lateral hydraulic sealing devices of the fracture surface are installed on both sides of the box, and the intake is connected to the fracture hydraulic device directly through the distributive pipe in order to control the hydraulic sealing and to realize the real-time monitoring of the hydraulic pressure to achieve precise control of the sealing cranny hydraulic pressure through computers.

The finished test pieces are combined and assembled accurately and sealed in a seepage box by a seal ring. After the assembly of the test piece, the cavity will be injected with liquid plastic of a certain pressure, and the seal ring under pressure is closely connected to the surrounding of the test piece; thus, the sealing of the test piece and the 
TABLE 1: The specification and mixture ratio of fracture production material.

\begin{tabular}{|c|c|c|c|c|c|c|}
\hline Material & Cement & & Particle size of sand (mm) & & Water & Water reducer \\
\hline Specification & $\begin{array}{l}\text { P.O } \\
42.5\end{array}$ & $\begin{array}{c}\text { Coarse } \\
0.8\end{array}$ & $\begin{array}{c}\text { Medium } \\
0.4\end{array}$ & $\begin{array}{c}\text { Fine } \\
0.2\end{array}$ & Common & DC-WR1 \\
\hline Ratio & 0.9 & & 1.8 & & 0.36 & 0.0135 \\
\hline
\end{tabular}

TABLE 2: The parameter table of permeability coefficient calculation.

\begin{tabular}{lcccc}
\hline $\begin{array}{l}\text { Gravitational acceleration } \\
G /\left(\mathrm{m} / \mathrm{s}^{2}\right)\end{array}$ & $\begin{array}{c}\text { Penetrating length } \\
L /(\mathrm{m})\end{array}$ & $\begin{array}{c}\text { Width of fracture } \\
w /(\mathrm{m})\end{array}$ & $\begin{array}{c}\text { Unit weight of water } \\
\gamma_{\omega} /\left(\mathrm{n} / \mathrm{m}^{3}\right)\end{array}$ & $\begin{array}{c}\text { Dynamic viscosity of water } \\
\nu /\left(\mathrm{m}^{2} / \mathrm{s}\right)\end{array}$ \\
\hline 9.81 & 0.2 & 0.1 & $1 e 4$ & $1.14 e-6$ \\
\hline
\end{tabular}

seepage box is realized; at the same time, contact surfaces of the upper seal ring and the lower seal ring are closely contacted under the pressure. The schematic diagram and the site picture are shown in Figure 3. During the experiment, the lateral hydraulic pressure is applied to the seepage box to effectively block the fracture part of the anastomotic rock mass. Therefore, the lateral complete sealing of the fracture surface is realized in the seepage process, and it is for sure that the direction of seepage only takes along the established fracture surface.

3.3. Measurement of the Roughness of Fracture Surfaces. For the analysis and research of the influence of microroughness on the seepage ability, the laser tester of the jointed rock surface and shape produced by the KEYENCE Company is applied to measure the surface shape of the joint (JRC) after the generation of the test piece, of which the accuracy is $\pm 20 \mu \mathrm{m}$ and the resolution is $10 \mu \mathrm{m}$. During the laser scanning, the laser head remains stationary and the platform on which the test piece is placed will move automatically in the $X Y$ direction according to the preset path; meanwhile, a computer is used to collect and process the data in real time. In the study, the distance between the measuring points on the surface of all rock test pieces are all $0.2 \mathrm{~mm}$ in the $X Y$ direction.

3.4. Preparation and Settlement of the Test Pieces. The artificial single fracture rock mass is used as the test piece, of which the specification is the $100 \mathrm{~mm}$ (width) $* 100 \mathrm{~mm}$ (height) * $200 \mathrm{~mm}$ (length) cuboid; the group that the Barton roughness coefficient JRC $=6-8$ recommended by ISRM is selected for the fracture specification; the numerical control technology is used to process the corresponding steel template, and then the fracture surfaces are made by assembling the steel template and the molds.

Three kinds of specifications sand of the particle size of $0.8 \mathrm{~mm}, 0.4 \mathrm{~mm}$, and $0.2 \mathrm{~mm}$ by the standard screen of the laboratory are prepared, and the P.O 42.5 cement, sand of different particles, and the benzene high efficiency water reducing agent DC-WR1 are chosen as the materials of the test pieces, of which the ratio is shown in Table 1.

The mold was demolished after the test pieces were made for 24 hours, and they stay at the curing room of standard test pieces where the temperature is $20 \pm 2^{\circ} \mathrm{C}$ and the relative humidity is over $95 \%$ for 28 days. The manufacturing and maintenance of test pieces are shown in Figure 4.

After the manufacturing and maintenance, the fracture surface morphology of different particle size is shown in Figure 5.

3.5. Test Scheme and Process. Before starting the test, firstly, the assembled seepage box is installed to the system platform by the lifting appliance, and the water seal confining pressure system of the seepage box is connected to the hydraulic loading system; debugging the computer control system, it is necessary to start the test when system shows normal. At first, a normal load is applied to the seepage box to reach a predetermined value; then, water seal pressure $(2.5 \mathrm{MPa})$ is applied to reach a steady state; at last, after applying the cranny hydraulic pressure and getting the corresponding data, the next test is proceeded to be done.

The test sets four groups of different normal load of 0.5 MPa, 1.0 MPa, 1.5 MPa, and 2.0 MPa, and the loading is implemented step by step in the condition that the loading rate is $0.1 \mathrm{~mm} / \mathrm{min}$; meanwhile, under the action of the normal load of each group, the seepage pressure of 0.2 $\mathrm{MPa}, 0.4 \mathrm{MPa}, 0.6 \mathrm{MPa}, 0.8 \mathrm{MPa}$, and $1.0 \mathrm{MPa}$ is added, respectively, and the water seal confining pressure is kept invariant during the whole test process.

After the test, firstly, the cranny hydraulic pressure is removed, then the water seal confining pressure, and the axial load finally.

\section{Test Results and Analysis}

4.1. Test Results. Formula (18) is used to calculate the permeability coefficient, and the corresponding parameters are presented in Table 2, of which the seepage length and fracture width are from the size of the sample, and the dynamic viscosity of water calculation parameter is from the reference [25] when the water temperature is $15^{\circ} \mathrm{C}$. After substituting the parameters, the formula of permeability coefficient can be simplified into

$$
k_{f}=6.593 e 6 \sqrt[3]{\frac{q^{2}}{\Delta p^{2}}}(\mathrm{~cm} / \mathrm{s})
$$



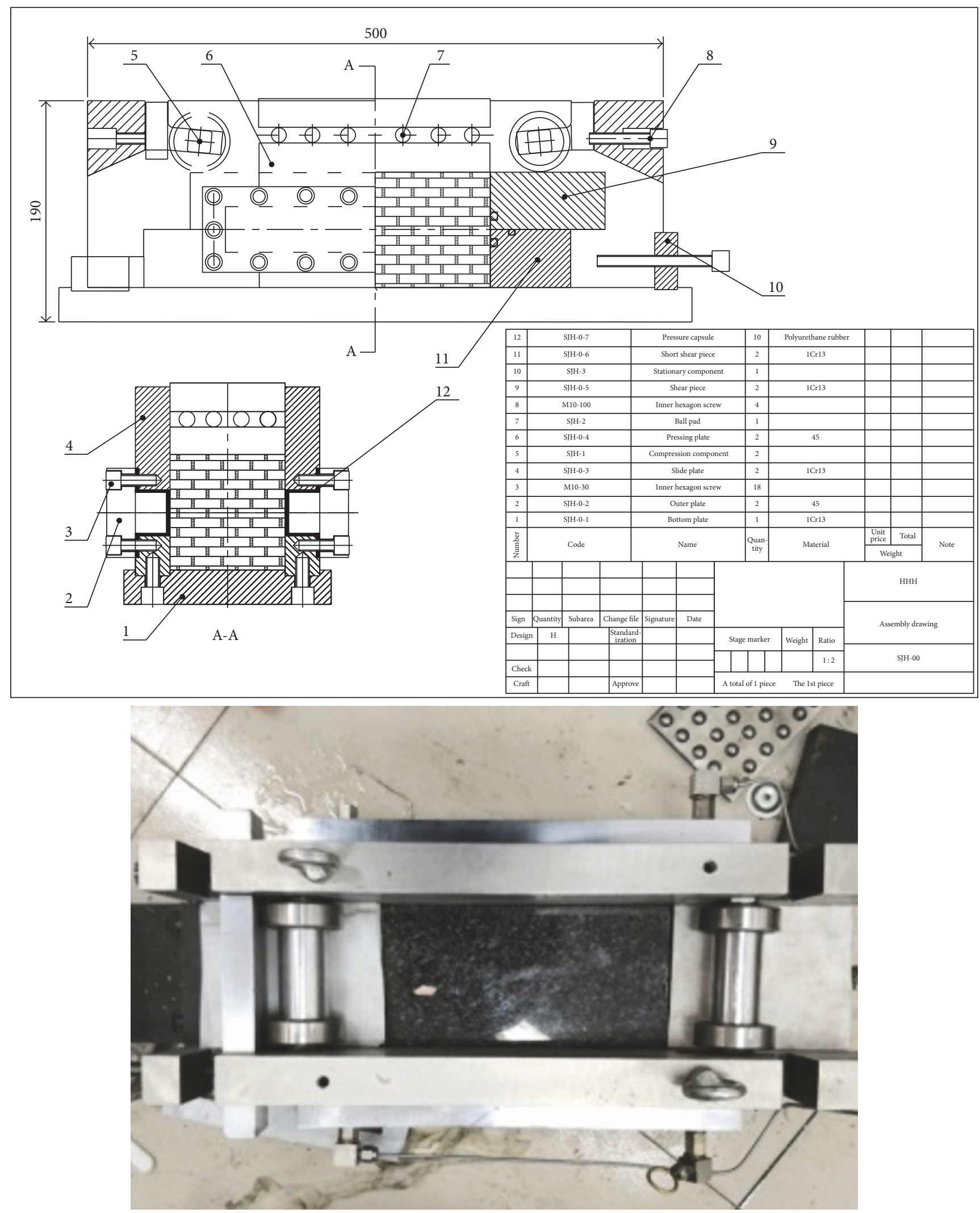

Figure 3: The seepage box assembly and its diagram. 

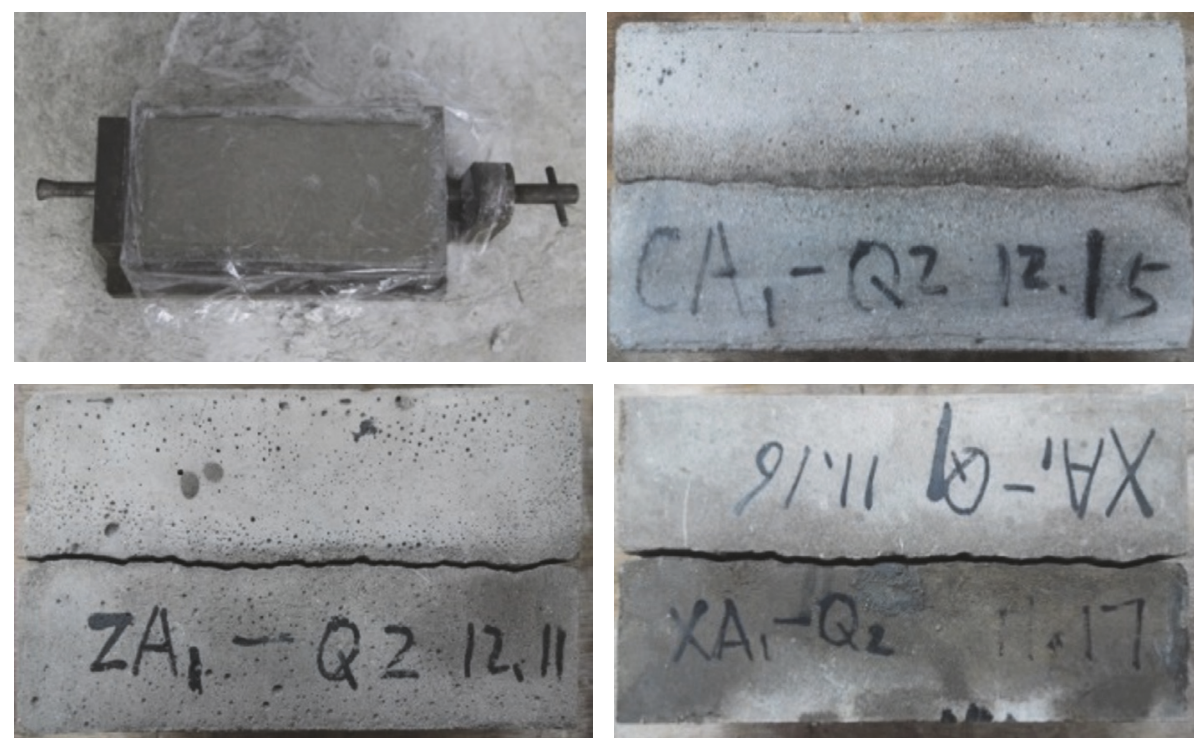

FIGURE 4: The process of manufacture and maintenance for quasi-sandstone body crack test pieces.

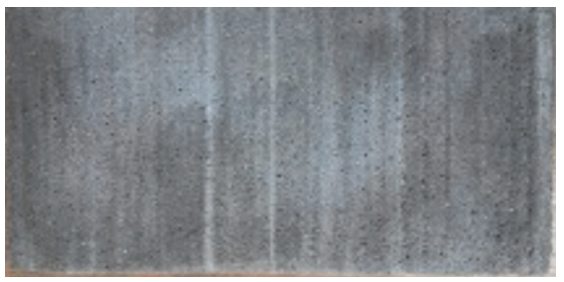

(a)

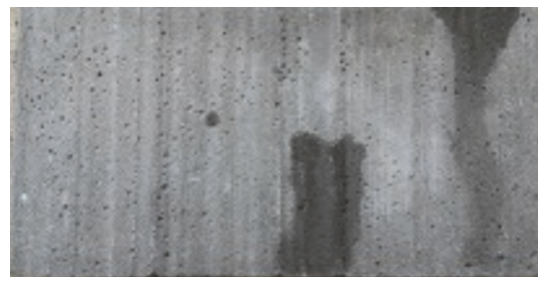

(b)

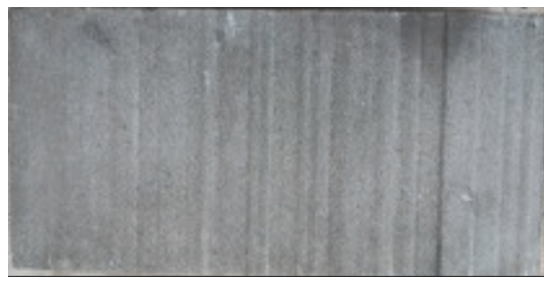

(c)

FIGURE 5: The fracture surface morphology of different particle size (from (a) to (c): coarse, medium, and thin).

4.2. Variation Law between Permeability Coefficient and Cranny Hydraulic Pressure. The data of seepage flow and the difference of seepage pressure of each group obtained in the test is substituted into formula (19); after solving the formula, the value of permeability coefficient of each group in the test is obtained; inducing and analyzing the result, the obtained relation curve between the permeability coefficient and the cranny hydraulic pressure is shown in Figure 6.

Through the analysis of Figure 6, it is found that for the fracture surfaces of various particle size, under the same normal load level, the permeability coefficient and the cranny hydraulic pressure basically present nonlinear relationship. It indicates that with the increase of the cranny hydraulic pressure, the permeability coefficient shows the exponential growth approximately. Besides, with the increase of the normal stress level, the growth rate is decreasing gradually, and the collectively presented variation law of permeability coefficient among different particle size is the fracture of coarse sand greater than the fracture of medium sand greater than the fracture of fine sand. It is concluded that the inlet of the cranny hydraulic pressure creates the compression effect on the fracture surface under the condition of constant normal stress and causes the additional deformation of the rock on both sides of the fracture surface, leading to the increasing of the fracture aperture and the permeability coefficient.

4.3. Variation Law between Permeability Coefficient and Normal Load. For the same cranny hydraulic pressure, the permeability coefficient is basically in accordance with the normal load variation. Therefore, taking the case that the cranny hydraulic pressure is $0.5 \mathrm{MPa}$ as an example, the variation relationships between different fracture microroughness and the normal loads are studied, as shown in Figure 7.

It can be seen from the figure that under the same cranny hydraulic pressure $(0.5 \mathrm{MPa})$, with the increasing of the normal load, the permeability coefficient of the fracture of different particle size shows a decreasing power function approximately. At the same time, under the same normal load, the permeability coefficients of different particle-sized fractures are different. It indicates that the smaller the particle size, the smaller the permeability coefficient; as the particle size increases, the permeability coefficient increases. The reason is that, under the same normal load, the fracture surface is compressed and deformed, but due to its different microparticle and combining structure, the particle size is 

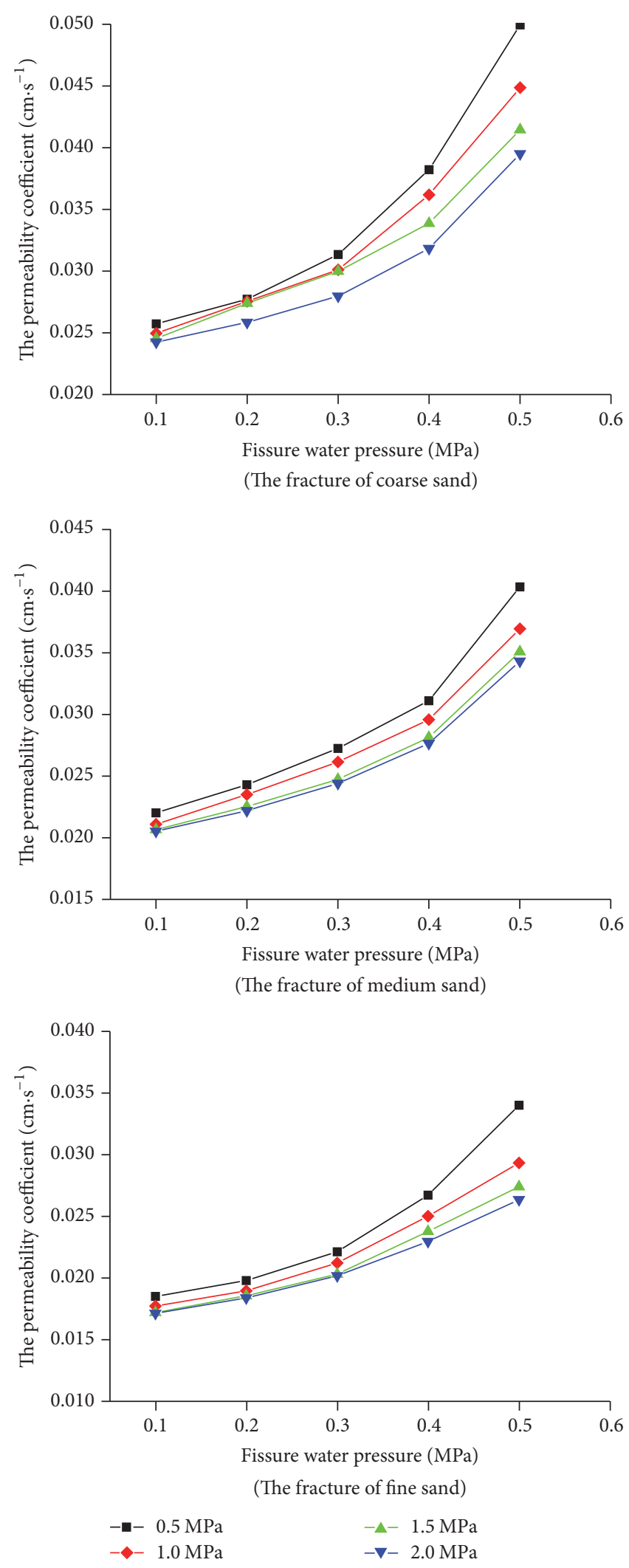

FIgURE 6: The rule curve between permeability coefficient and cranny hydraulic pressure. 


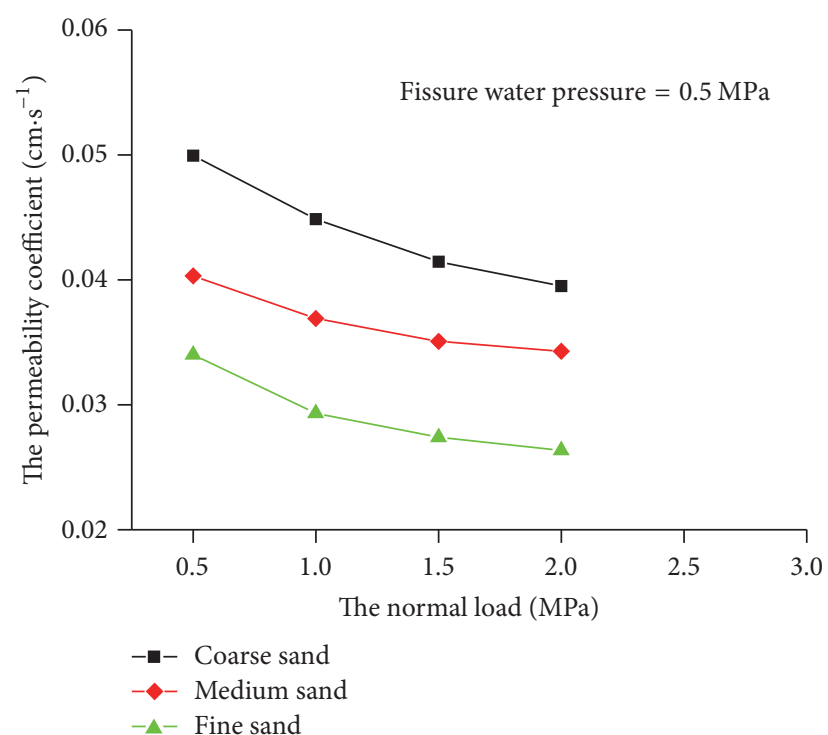

Figure 7: The rule curve between permeability coefficients and normal loads.

bigger; the interspace generated between fracture surfaces becomes bigger; the hindering effect on the water flow becomes smaller; thus the permeability coefficient becomes bigger. On the contrary, the smaller the particle size, the smaller the interspace between the fracture surfaces; under the action of the load, the goodness of fit of the fracture surface becomes higher, the hindering effect on the water flow becomes bigger, and thus the permeability coefficient becomes smaller. It can be found, therefore, that for the same JRC roughness coefficient curve, under the same external environment, the seepage capability of the fracture surfaces of different particle size is different.

The microscopic roughness mainly reduces the energy of the water flow caused by the viscous effect and adsorption of water flowing through the fracture surface, which results from the influence of small particle size distribution, particle size, and its different combination forms. However, the macroscopic roughness dissipates the energy of water mainly dependent on the wavy elevation difference of fracture surface to affect the seepage ability of water.

4.4. Solving of the Microroughness Coefficient. In order to ensure the accuracy of the solution of the microroughness coefficient and reduce the influence of the JRC on the permeability coefficient, the surface roughness of the test piece is tested by the laser tester of the jointed rock surface and shape. The Z2 calculation method [32,33] was adopted in this paper, coarse, medium, and fine fractures with different particle diameters were calculated, and the JRC values of the surface roughness were 7.5, 7.8, and 7.2, respectively.

The stress and the strain values of the coarse particlesized test piece are obtained from the laboratory test; $E_{t}=$ $6325 \mathrm{MPa}$ and $E_{r}=6517 \mathrm{MPa}$ are obtained through the fitting operation; $\mu_{0}=54 \mu \mathrm{m}$ is obtained after the measurement. Programming formula (15) by the FISH language built-in ITASCA and substituting the relating parameters to get the

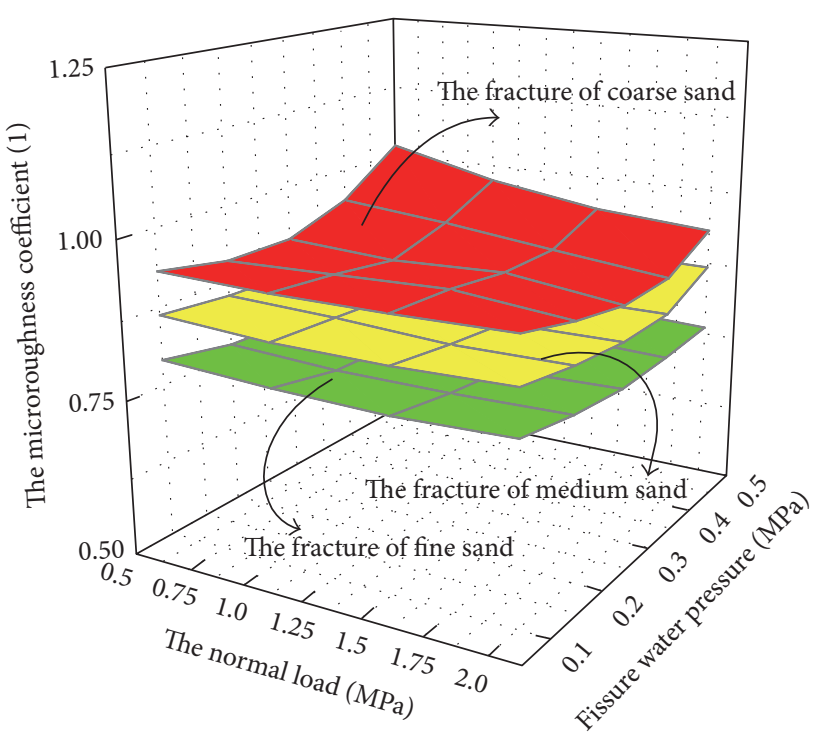

FIGURE 8: The 3D plane of microroughness coefficient.

corresponding theoretical calculated value, the comparison and solution are carried out between it and the measured value. For the medium and the fine particle-sized test pieces, the corresponding values of $E_{t}, E_{r}$, and $\mu_{0}$ are obtained by the same method, and the programming calculation is used to get the microroughness coefficients of different particle size. The normal load (0.5-2.0 MPa) and the cranny hydraulic pressure (0.1-0.5 $\mathrm{MPa})$ of each group, respectively, correspond to a microroughness coefficient value; thus all the microroughness coefficients of the fracture of each particle size are approximately manifested as a plane in the graph, and the results are shown in Figure 8.

The average value of the microroughness coefficient of each fracture surface is calculated, and the average values of the coarse, medium, and thin particle-sized fractures are, respectively, $0.772,0.851$, and 0.926 . It characterized that as the particle sizes become bigger, the microroughness coefficients increase gradually with the amplifications of $10.2 \%$ and $8.8 \%$, and the permeability coefficients also increase. It can be seen that, in the case of the same JRC, the corresponding permeability coefficient is different due to the different particle size and structural characteristics of the fracture surface. Therefore, the influence of the microroughness of the fracture surface on the seepage capability cannot be neglected.

4.5. Verification of the Theoretical Formula. Based on the microroughness coefficient of the fracture surfaces of different particle size obtained in the above analysis, the same test pieces as the former test are selected. Five groups' stressseepage coupling tests are done with the cranny hydraulic pressure being 0.1 to $0.5 \mathrm{MPa}$, respectively, for the two cases where the normal loads are $2.5 \mathrm{MPa}$ and $3.0 \mathrm{MPa}$ and the validity and accuracy of the microroughness coefficient are verified. The test results and the theoretical validation values considering the microroughness coefficient are shown in Figure 9. 

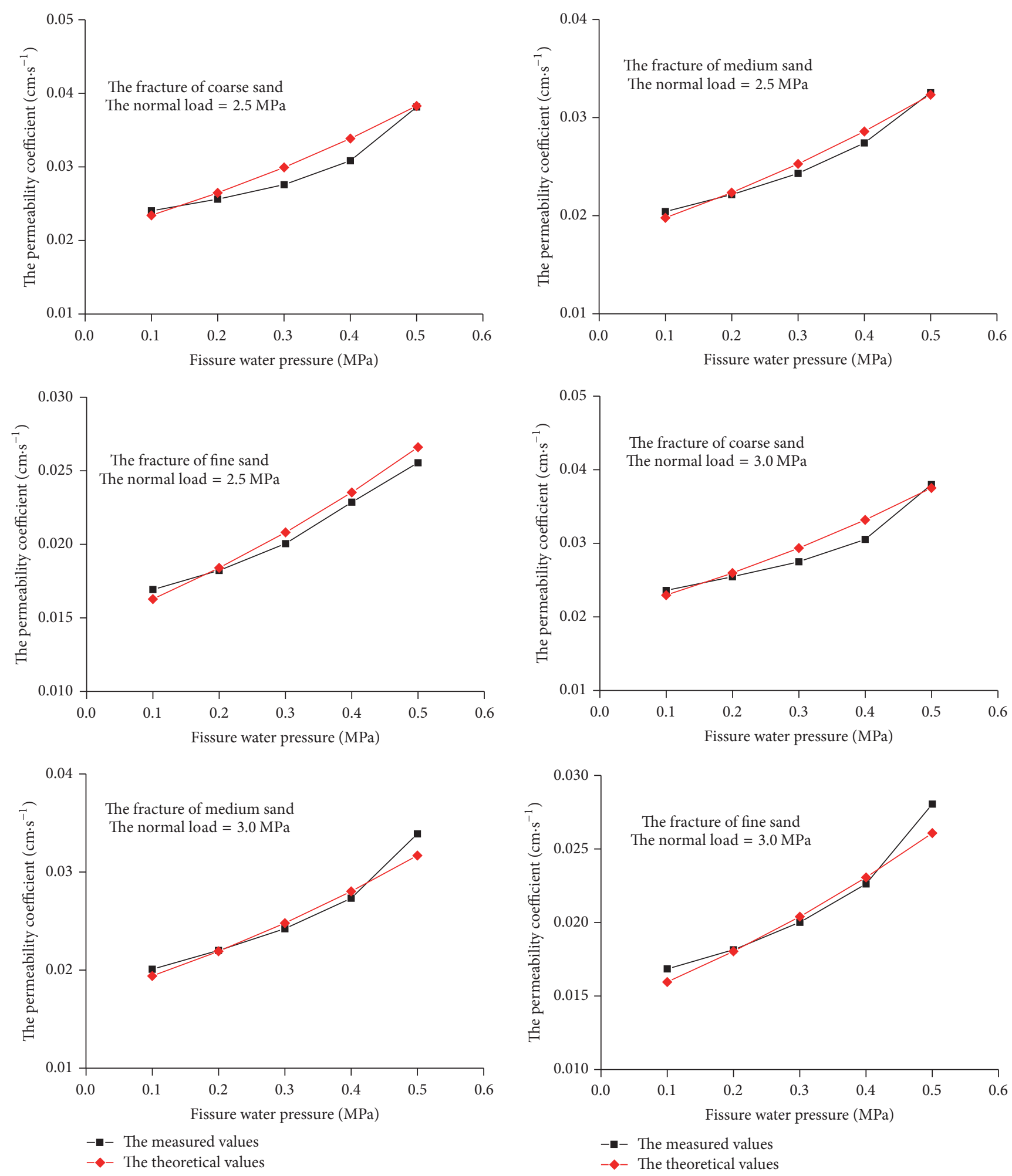

FIGURE 9: The comparison of permeability coefficient between the theoretical values considering the microroughness and the measured values.

As shown in the figure, when the normal loads are, respectively, 2.5 $\mathrm{MPa}$ and 3.0 $\mathrm{MPa}$, the goodness of fit between the theoretical validation values and the measured values of the permeability coefficient of different particle-sized fracture surface is higher, which means, through considering the microroughness coefficient introduced by the nature of the microsurface of the fracture, formula (15) is able to describe the variation law between the permeability coefficient and the 
fracture water surface and show the difference in the seepage capability of the different particle-sized fracture surface of the rock mass. In the case of the stress-seepage coupling of the fracture surface of the jointed rock mass, it is not comprehensive for the seepage description to only consider the JRC permeability of the fracture surface; therefore, the effect of the nature and the particle distribution of the microsurface of the fracture on the seepage capability are not negligible.

\section{Conclusion}

(1) Based on the results of the test among the joint roughness coefficient (JRC) of rock fracture, mechanical aperture, and hydraulic aperture proposed by Barton, the effect of different particle size and its structure on the seepage capability is considered under the same JRC condition by introducing a microroughness coefficient, and the permeability coefficient formula of single fracture surfaces considering the microroughness is deduced under the condition of stressseepage coupling, of which the parameter can be calculated through the indoor test with the feasibility and general applicability.

(2) Through the indoor test, manufacturing the molds, and preparing the fracture surface of different particle size, stress-seepage coupling tests are carried out, respectively, under different normal load and different seepage pressure to get the permeability coefficient of different particle-sized fracture surfaces; through the analysis of the test results, it is found that, in the case of the same JRC, the seepage pressure, and the normal load, the seepage capability of different particlesized fracture surface is different. With the increase of the particle size, the permeability coefficient of the fracture surface increases correspondingly; the smaller the particle size, the smaller the permeability coefficient. It shows that the effect of microroughness of the fracture surfaces on its seepage capability cannot be neglected.

(3) Under the same lower normal stress level, with the increasing of cranny hydraulic pressure, permeability coefficients of deferent particle-sized fractures show the exponential growth, while for the same cranny hydraulic pressure, permeability coefficients of different particle-sized fractures approximately show a decreasing power function with the normal load.

(4) The FISH language is used to solve the formula of the single fracture stress-seepage coupling considering microroughness by programming in order to get the microroughness coefficients of fracture surfaces of different particle size. The correctness and validity of the two parallel seepage tests are verified under different normal load level, and the result shows better goodness of fit. At the same time, it also indicates that the theoretical formula of single fracture stressseepage coupling deduced by the introduction of the microroughness coefficient is reasonable and reliable.

\section{Conflicts of Interest}

The authors declare that there are no conflicts of interest regarding the publication of this paper.

\section{Acknowledgments}

The authors would like to express appreciation to Dr. Gang Wang and doctoral student Weijie Song at Shandong University of Science and Technology for their experimental assistance. This project is financially supported by the National Natural Science Foundation of China, Grants nos. 51474135 and 41272325 .

\section{References}

[1] Y. Jiang, B. Li, G. Wang, and S. Li, "New advances in experimental study on seepage characteristics of rock fractures," Yanshilixue Yu Gongcheng Xuebao/Chinese Journal of Rock Mechanics and Engineering, vol. 27, no. 12, pp. 2377-2386, 2008.

[2] S. C. Bandis, A. C. Lumsden, and N. R. Barton, "Fundamentals of rock joint deformation," International Journal of Rock Mechanics and Mining Sciences \& Geomechanics Abstracts, vol. 20, no. 6, pp. 249-268, 1983.

[3] R. E. Goodman, Methods of Geological Engineering in Discontinuous Rocks, West Publishing Company, NY, USA, 1976.

[4] G. Sun and W. Lin, "The Compresstional Deformation Law of Rock Mass Structure Surface and a Constitutive Equation of Rock Mass Elastic Deformation," Chinese Journal of Geology, no. 2, pp. 177-180, 1983.

[5] G. Sun, Rock Mass Structural Mechanics, Science Press, Beijing, China, 1988.

[6] N. Barton and V. Choubey, "The shear strength of rock joints in theory and practice," Rock Mechanics Felsmechanik Mécanique des Roches, vol. 10, no. 1-2, pp. 1-54, 1977.

[7] N. Barton, "Modelling rock joint behavior from in situ block tests: Implications for nuclear waste repository design," Tech. Rep., Office of Nuclear Waste Isolation, Columbus, OH, USA, 1982.

[8] G. M. Lomize, Flow in Fractured Rocks, Gosenergoizdat, Moscow, Russia, 1951.

[9] E. S. Romm, Flow Characteristics of Fractured Rocks, Nedra, Moscow, Russia, 1966.

[10] D. T. Snow, "Anisotropie permeability of fractured media," Water Resources Research, vol. 5, no. 6, pp. 1273-1289, 1969.

[11] Z. Lu, J. Yao, D. Wang, and L. Li, "Correction of cubic law and calculation of critical velocity in parallel fractures," Research and Exploration in Laboratory, vol. 29, no. 4, pp. 14-17, 2010.

[12] Z. Wang, L. Shen, S. Li, and Z. Xu, "Seepage characteristics of a single fracture based on lattice Boltzmann method," Rock and Soil Mechanics, vol. 38, no. 4, pp. 1203-1210, 2017.

[13] C. Louis and Y. N. T. Maini, "Determination of in situ hydraulic parameters in jointed rock," in Proceedings of the 2nd ISRM Congress, 1970.

[14] B. Amadei and T. A. Illangasekare, "Mathematical model for flow and solute transport in nonhomogeneous rock fracture," International Journal of Rock Mechanics and Mining Sciences \& Geomechanics Abstracts, vol. 18, pp. 719-731, 1994.

[15] B. Su, M. Zhan, and J. Zhao, "Study on Fracture Seepage in the Imitation of natural rock mass," Chinese Journal of Geographical Engineering, vol. 05, pp. 19-24, 1995. 
[16] K. Geng, F. Chen, G. Liu, and X. Chen, "Experimental research of hydraulic properties of seepage flow in fracture," Qinghua Daxue Xuebao/Journal of Tsinghua University, vol. 36, no. 1, pp. 102-106, 1996.

[17] C. Zhou and W. Xiong, "A generalized cubic law for seepage in rock joints," Rock and Soil Mechanics, vol. 04, pp. 1-7, 1996.

[18] Y. He, Y. Tao, and L. Yang, "Experimental research on seepage behaviors in single fracture with various values of JRC," Chinese Journal of Rock Mechanics and Engineering, vol. 29, no. zl, pp. 3235-3241, 2010.

[19] H. Yu, F. Chen, W. Chen, J. Yang, J. Cao, and K. Yuan, "Research on permeability of fractured rock," Yanshilixue Yu Gongcheng Xuebao/Chinese Journal of Rock Mechanics and Engineering, vol. 31, no. 1, pp. 2788-2795, 2012.

[20] Z. Chang, Y. Zhao, Y. Hu, and D. Yang, "Theoretic and experimental studies on seepage law of single fracture under 3D stresses," Chinese Journal of Rock Mechanics and Engineering, vol. 23, no. 4, pp. 620-624, 2004.

[21] Y. Zeng, D. Lu, and Q. Zeng, "Experimental study on coupling of flow-stress with in a single fracture," Journal of Experimental Mechanics, vol. 20, no. 1, pp. 10-16, 2005.

[22] C. Liu and C. Chen, "The seepage characteristics of single fractured rock under triaxial stress," Progress in Natural Science, vol. 17, no. 7, pp. 989-994, 2007.

[23] L. Xu and N. Xie, "Test and theoretical research on permeability characteristics of shear fracture in rock mass," Yanshilixue Yu Gongcheng Xuebao/Chinese Journal of Rock Mechanics and Engineering, vol. 28, no. 11, pp. 2249-2257, 2009.

[24] S. Zheng, Y. Zhao, and K. Duan, "An Experimental Study on The Permeability Lawof Natural Fracture Under 3-D Stresses," Chinese Journal of Rock Mechanics and Engineering, vol. 02, pp. 15-8, 1999.

[25] G. Xu, Y. Zhang, and Q. Ha, "Experimental study on hypercube and the cubic law of rough fracture seepage," Journal of Hydraulic Engineering, vol. 03, pp. 74-79, 2003.

[26] J. Liu, "Seepage equation of single fracture under normal stress," Hydrogeology and Engineering Geology, vol. 02, pp. 32-33, 1987.

[27] J. Liu, "The coupled relationships between mechanical and hydraulic parameters of a structural surface and their applications," Hydrogeology and Engineering Geology, vol. 02, pp. 7-12, 1988.

[28] S. Sha and G. Zhang, "Modeling of hydraulic fracture of concrete gravity dams by stress-seepage-damage coupling model," Mathematical Problems in Engineering, Article ID 8523213, 15 pages, 2017.

[29] T. Yang, P. Wang, H. Liu, T. Xu, Q. Yu, and W. Shi, "Deformational behavior of underground opening using a stressseepage coupled model considering anisotropic characteristics," Arabian Journal of Geosciences, vol. 8, no. 9, pp. 6635-6642, 2014.

[30] T. Nie, H. Pu, G. Liu, C. Xiao, and H. Ni, "Research on splitting failure model of fractured rock mass under the coupling effect of seepage-stress," Caikuang yu Anquan Gongcheng Xuebao/Journal of Mining and Safety Engineering, vol. 32, no. 6, pp. 1026-1036, 2015.

[31] Y. Tao, An experimental study on hydraulic behavior in a single joint with different JRC, Southwest Jiaotong University, 2007.

[32] H.-Q. Sun and H.-P. Xie, "Fractal simulation of rock fracture surface," Yantu Lixue/Rock and Soil Mechanics, vol. 29, no. 2, pp. 347-352, 2008.
[33] Q.-Y. Wang and Y. Li, "Experimental study of scale effects in strength and deformation of fractal-joint rock masses," Yantu Lixue/Rock and Soil Mechanics, vol. 29, no. 5, pp. 1325-1328, 2008. 


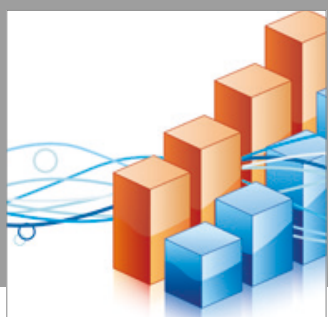

Advances in

Operations Research

vatersals

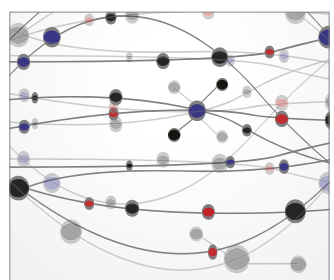

\section{The Scientific} World Journal
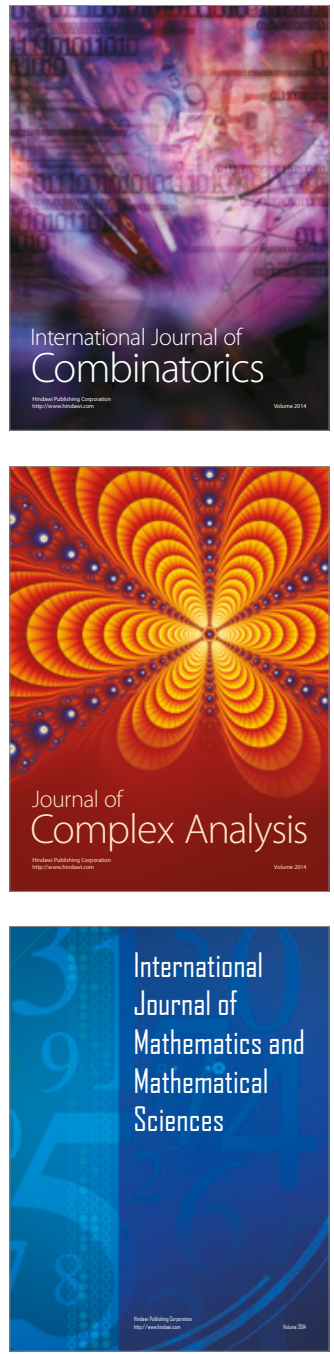
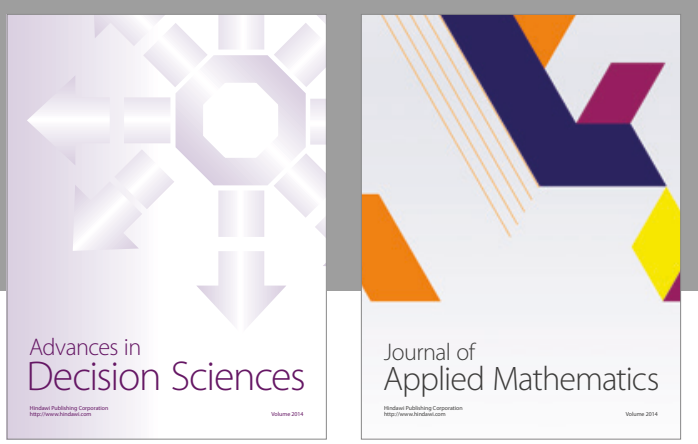

Algebra

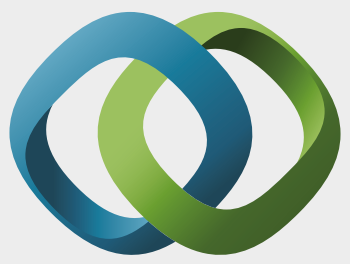

\section{Hindawi}

Submit your manuscripts at

https://www.hindawi.com
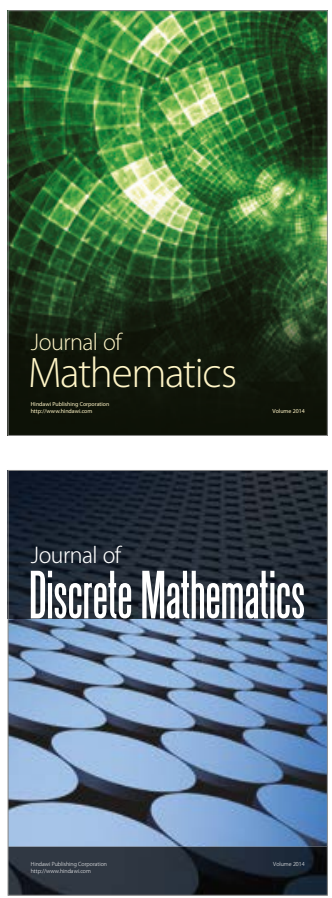

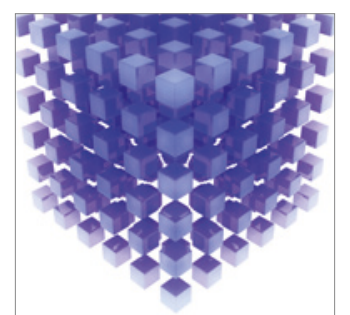

Mathematical Problems in Engineering
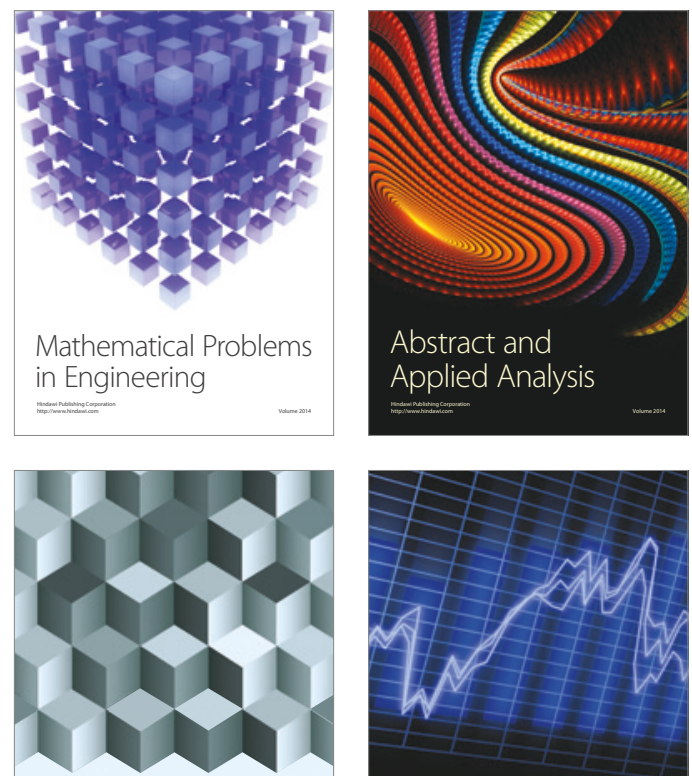

Journal of

Function Spaces

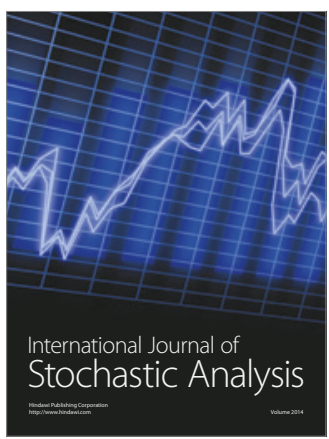

Probability and Statistics
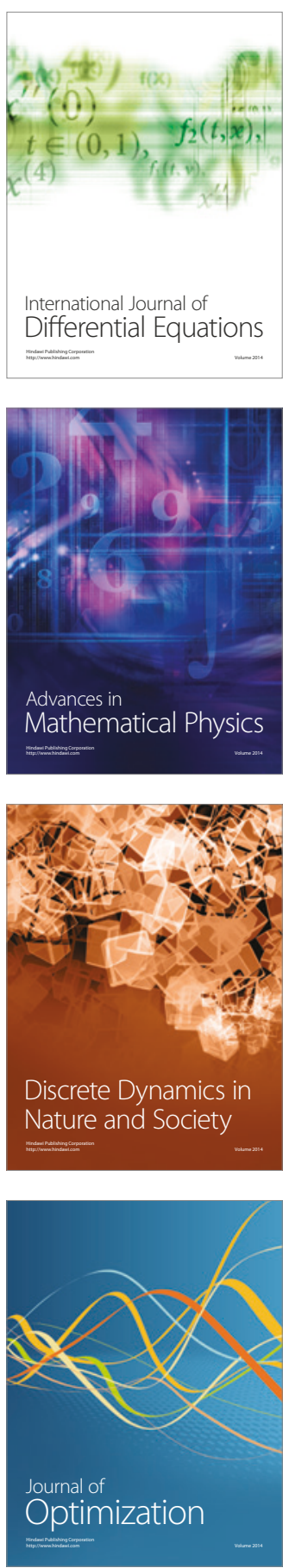